\title{
Surrogacy in invasion research and management: inferring "impact" from "invasiveness"
}

\author{
Luke S O’Loughlin ${ }^{1,2,3 *}$, Ben Gooden ${ }^{4,5}$, Jacob N Barney ${ }^{6}$, and David B Lindenmayer ${ }^{1}$
}

Biological invasions are known drivers of biodiversity decline, yet the ecological impacts of invaders remain largely unmeasured in many contexts. Consequently, other measures of a species invasion (eg local abundance) are often used as surrogates (or "proxies") to infer impact on recipient ecosystems. However, the use of surrogates for impact in invasion science and management is often implicit, and frequently lacks the evaluation and validation that characterize surrogate use in other fields. Although there are practical reasons for this, the risks associated with not testing the accuracy, stability, and certainty of surrogates for invasive species impact must be acknowledged. Recognizing the role of surrogacy in invasion science offers previously unappreciated solutions for increasing the quantitative rigor of invasive species impact assessments that inform management decisions.

Front Ecol Environ 2019; 17(8):464-473, doi:10.1002/fee.2097

Tnvasive alien species represent major threats to biodiversity 1 through their impacts on recipient ecosystems (Courchamp et al. 2017). Despite this, the ecological consequences associated with the success of most alien species remain poorly explored and are seldom quantified (Barney et al. 2013; Blackburn et al.

\section{In a nutshell:}

- Invasive species pose major threats to native species and ecosystems, yet their impacts remain poorly explored and are rarely quantified

- There is a practical need to identify invasive species and prioritize their management based on the magnitude of their impact, meaning easier-to-measure variables (such as an invasive species' local abundance) are widely used to infer unmeasured impacts

- This practice of inferring invasive species impacts from surrogate measures is widespread in invasion science and management, but often lacks the requisite evaluation and validation that is commonplace in other disciplines where surrogates are used

- Greater integration of ideas from surrogate research into invasion ecology offers a previously unrecognized solution to issues related to quantifying, demonstrating, managing, and communicating the ecological impacts of invasive species

\footnotetext{
${ }^{1}$ Fenner School of Environment and Society, The Australian National University, Canberra, Australia; ${ }^{2}$ School of Environmental Science, Charles Sturt University, Albury, Australia; ${ }^{3}$ Current address: Department of Ecology, Environment and Evolution, La Trobe University, Bundoora, Australia ${ }^{*}$ L.OLoughlin@latrobe.edu.au); ${ }^{4}$ CSIRO Health and Biosecurity, Canberra, Australia; ${ }^{5}$ School of Earth, Atmospheric and Life Sciences, Faculty of Science, Medicine and Health, University of Wollongong, Wollongong, Australia; ${ }^{6}$ School of Plant and Environmental

Sciences, Virginia Tech, Blacksburg, VA
}

2014). Demonstrating and predicting the impacts of invasive species on recipient ecosystems is difficult due to the highly context-specific interactions and drivers that characterize most invasions (Courchamp et al. 2017; O'Loughlin and Green 2017b). Closing this knowledge gap represents an ongoing challenge for invasion research and management. Acknowledging this, many researchers have proposed useful approaches for improving direct assessments of impact, including semi-quantitative categorical frameworks (eg Blackburn et al. 2014), quantitative metrics that integrate multiple measured effects (eg Barney et al. 2013), and predictive tools that use explicit measures of a species' per capita effects (eg the functional [consumption] and numerical [abundance] response of a predator at a particular density of prey; Dick et al. 2017). However, direct assessments of impact often do not occur, with researchers and practitioners instead relying on surrogate measures to infer unmeasured effects of invasive species on native ecosystems, and inform the prioritization and management of such invaders (Kumschick et al. 2012).

Measuring a surrogate (or "proxy" or "indicator") for a target of interest for which direct measurement is difficult is common practice across a broad range of disciplines, from medical research to fundamental ecology and natural resource management (O'Loughlin et al. 2018). In ecology, the usual approach is to measure one variable in the ecosystem (eg plant species richness) and use the measurement(s) to infer something unmeasured (eg ecosystem productivity) based on a known relationship, established at an earlier point in time, between the surrogate and the target (Lindenmayer et al. 2015). Similar to concepts like biodiversity, the ecological impacts of an alien species are often too complex to be assessed completely (Jeschke et al. 2014). Consequently, any meaningful conclusions regarding invader impacts will depend on the surrogate measures used to infer them (Hulme et al. 2013).

Because measuring effects through surrogates instead of directly measuring native ecosystem responses (ie the target) 
necessitates accepting a degree of uncertainty (regarding whether the chosen surrogate is suitable for inferring those effects), the use of surrogates sometimes leads to unwarranted or unqualified inferences (O'Loughlin et al. 2018). Relying on surrogates in the assessment of invasive species impacts is not explicitly recognized or discussed in invasion research or management. Therefore, most of the uncertainty and risk associated with how accurately a surrogate represents the ecological impacts of an invader is underappreciated. For example, the abundance of an invader is regularly used to infer unmeasured impacts on the recipient ecosystem (Pearson et al. 2016; Essl et al. 2017) because the invader's impact is, in part, a function of its density (Parker et al. 1999; Sofaer et al. 2018). However, widespread application of this logic to any alien species classified as "invasive" in any recipient ecosystem ignores the context sensitivity of the invasiveness-impact relationship (Barney et al. 2013; Essl et al. 2017), and does not account for per capita effects (Spencer et al. 2016; Dick et al. 2017; Pearse et al. 2019). Assuming that a species invasion affects multiple systems in the same way may oversimply the invasiveness-impact relationship and create false impressions of certainty (Johnson and Lidström 2018). In invasion science and policy, complexities and uncertainties must be explicitly and clearly understood and acknowledged - that is, the strength and confidence (in the statistical sense; that is, with respect to explanatory power and the variability/error in correlations) of surrogate-impact relationships should be evaluated and validated in a rigorous and systematic way. And yet such assessments are rare.

We discuss how the use of ecological surrogacy to infer unmeasured ecological impacts of invasive species is often overlooked in invasion science. This is not a critique of the many approaches for measuring ecological impact directly (eg Vilà et al. 2018) but rather a discussion of how surrogate measurements are used after a direct assessment is completed (or are used in place of a direct assessment) to infer impacts that remain unmeasured. We consider how both researchers and managers often justify the widespread application of a variety of surrogate-impact models based on limited, context-specific evidence of impacts involving a small number of invasive species. For simplicity, we restrict our discussion (for the most part) to ecological impacts, although we recognize that these surrogate measures may also be used to infer socioeconomic impacts, which are also important (Jeschke et al. 2014; Bacher et al. 2018). We also detail how greater integration of existing surrogate frameworks into invasion research and management offers a previously unrecognized solution to issues related to quantifying, demonstrating, managing, and communicating the ecological impacts of invasive species.

\section{Surrogate-impact models used in invasion research and management}

Using a surrogate is a practical solution for collecting meaningful information about a target of interest in cases where measuring that target directly or completely is too difficult as a result of financial, temporal, or logistical constraints (Lindenmayer et al. 2015; O'Loughlin et al. 2018). Surrogates are used in ecology to provide information about ecosystems ("indicator surrogates") and/or as tools to facilitate management intervention ("management surrogates") (Hunter et al. 2016). These two approaches are often complementary; for example, monitoring beaver abundance (Castor canadensis and Castor fiber) can act as an indicator surrogate by providing information on habitat availability for pond-dependent biota, and as a management surrogate by revealing whether management intervention to conserve beavers (eg predator control) has been successful (Hunter et al. 2016). Both forms of surrogacy are essential to invasion ecology, where the status of an invader can inform the most common research and management goals of (1) understanding the ecological impacts caused by invasive species (ie an indicator surrogate) and (2) effectively controlling those species as a means of conserving and enhancing native ecosystems (ie a management surrogate) (Courchamp et al. 2017).

Invasion itself can be conceptualized as two linked yet distinct components: the determinants and the consequences of invasion success. The combination of factors that facilitate invasion success have been thoroughly studied, leading to an array of proposed hypotheses that explain progress along the invasion pathway, and a detailed understanding of how measures of propagule pressure, species traits, and properties of recipient ecosystems can be used as surrogates to infer invasion success (Figure 1; Lockwood et al. 2005; Catford et al. 2009; Blackburn et al. 2011). As such, the use of surrogates to measure invasion success will not be detailed here. Conversely, the ecological consequences of invasions have been examined to a far lesser extent. The few hypotheses underpinning species impacts are not well supported by data, and confidence is lower in how measures of the invasiveness of a given species may act as surrogates for ecological impacts (Hulme et al. 2013; Essl et al. 2017). However, surrogates for impacts are widely used to identify which invasions will likely have the most severe ecological consequences and should be prioritized for management (Kumschick et al. 2012; Prior et al. 2018). In the sections below, we detail four key surrogate-impact relationships that underpin invasion research and guide invasive species management (Figure 1).

\section{(1) Invader presence as a surrogate for impact}

The presence of an invasive species is probably the simplest surrogate for impact used in both research and management (Figure 1, relationship 1), and is commonly employed where collecting robust data on local abundance or geographic extent may be prohibitive (eg a cryptic invader; Jarić et al. 2019). Using the presence of a species to infer its impact presupposes either (1) that impact is a fundamental characteristic of any alien species classified as "invasive" (Blackburn et al. 2014), and/or (2) that well-documented invasion-impact relationships can be extrapolated to invasive 


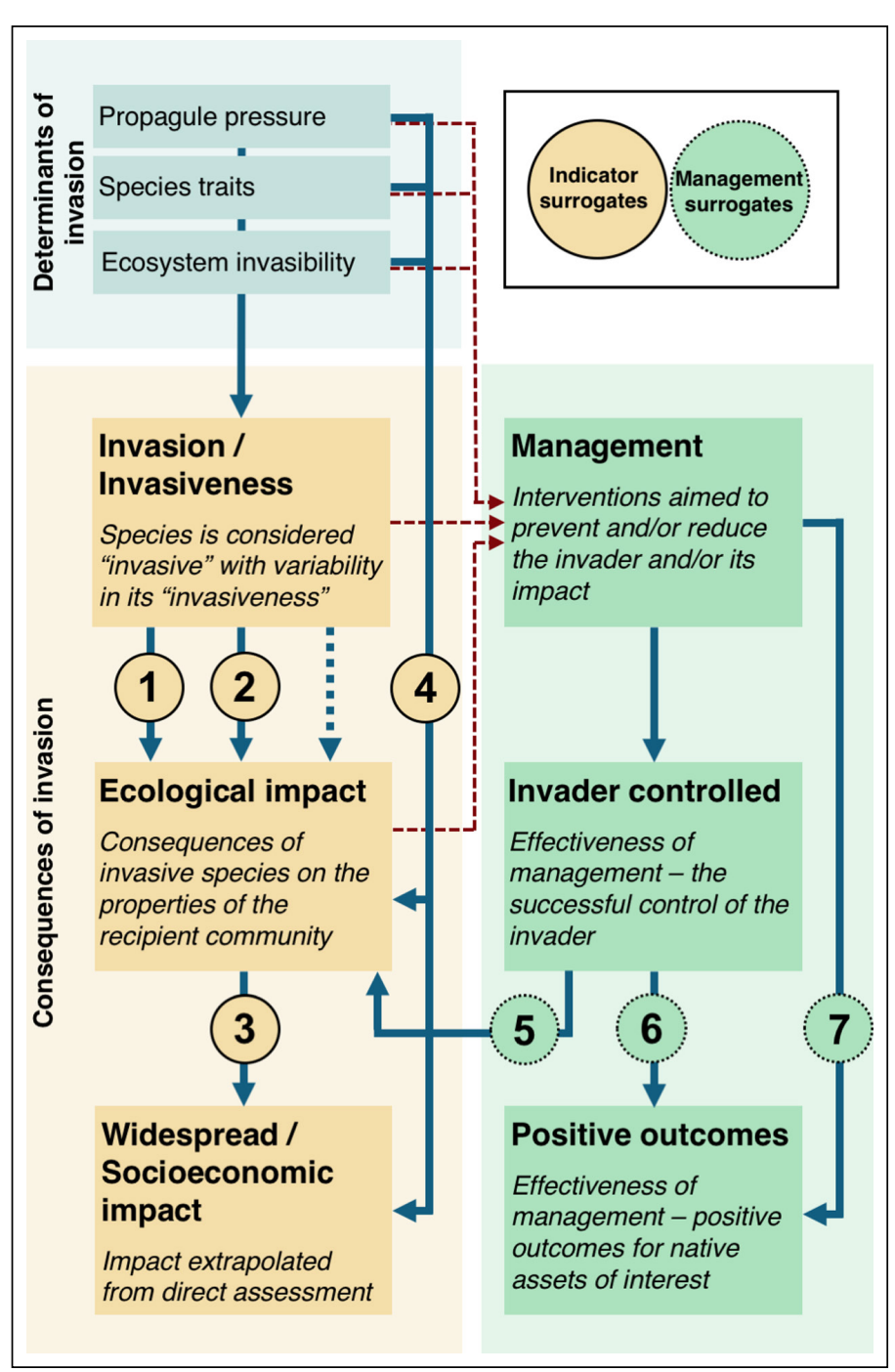

Figure 1. The surrogate models widely used in research and management to infer the determinants and consequences of species invasion. Arrows linking concepts reflect where measures of one are used to infer the other. Numbers refer to the surrogate-impact relationships that are either "indicator surrogates" ([1] presence is a surrogate for impact; [2] invasiveness is a surrogate for impact; [3] some impact is a surrogate for widespread impact; and [4] determinants of invasion are surrogates for consequences of invasion [ie ecological impact]) or "management surrogates" ([5] invader removal is a surrogate for removal of impacts; [6] invader removal is a surrogate for positive ecosystem outcomes; and [7] management intervention is a surrogate for positive ecosystem outcomes). Dashed red arrows reflect how measures of a species invasion are used to trigger invasive species management; dashed blue arrow reflects that there are other measures of a species "invasiveness" that we have not specifically detailed in the main text (eg geographic extent).

species whose impacts have not been measured (Barney et al. 2013; Nentwig et al. 2016). For example, this impact surrogate is often broadly applied to invasive plants, and justified by meta-analyses of published data that consistently reach the conclusion that invasive plants have negative effects on native communities (Figure 2a; Pyšek et al. 2012). However, these analyses are likely skewed by publication bias toward species, metrics, and conditions where the most substantial impacts would be expected (Didham et al. 2005; Guerin et al. 2018). Similarly, such analyses usually compare invaded and non-invaded areas but do not identify the threshold of invasion where impact would begin (Panetta and Gooden 2017). The evidence that supports presence as a robust surrogate for impact is often context dependent, and ignoring that context risks misrepresenting threats. Although presence-impact is a robust relationship for those highest-impact invaders (often referred to as "transformers" rather than "invasives"; Richardson et al. 2000), the presence of other invasive plants, for example, is just as likely to have neutral or positive ecosystem effects (Seabloom et al. 2013; Pearson et al. 2016).

\section{(2) Invasiveness as a surrogate for impact}

Measures of a species' invasiveness are widely considered to be an informative indicator of impact (Figure 1, relationship 2; Essl et al. 2017). There are multiple dimensions that contribute to the overall invasiveness of a species, including environmental range, geographic extent, rate of spread, and local abundance and density (Catford et al. 2016), all of which may relate to a species' impact. Density or abundance is probably the most common dimension of invasiveness used to infer impact, and is a relationship largely supported in individual studies (Parker et al. 1999; Barney et al. 2013). For example, Gooden et al. (2009) found considerable evidence of linear and non-linear impacts of increasing cover of an invasive shrub on native vegetation, suggesting invader abundance strongly represented its ecological effects (Figure 2b). However, it is well established that impact is also a function of per capita effects, which in turn are mediated by environmental factors independent of the invader; as such, invasiveness should not be expected to be a consistent surrogate for impact across time and space (Dick et al. 2017; O'Loughlin and Green 2017b; Sofaer et al. 2018). For instance, the only cross-taxonomic meta-analysis of the invasiveness-impact relationship (where rate of spread and establishment of the invader were the dimensions of invasiveness considered) found no consistent correlation in any biotic group (Ricciardi and Cohen 2007), and other reviews focusing on invasive plants have not considered this relationship, citing insufficient data (Pyšek et al. 2012; Catford et al. 2016). Despite the findings of these syntheses, the application of this surrogate (ie some dimension of invasiveness) in research and management is often justified by existing evidence of impact by the invader at some time and in some contexts (Kulhanek et al. 2011).

\section{(3) Some impact as a surrogate for widespread impact}

Measures of impact on some components of the recipient community in some contexts are often used to infer more widespread consequences of invasive species (Figure 1, 



Figure 2. Examples of species and contexts in which surrogate measures are often used to infer unmeasured ecological impacts: (a) in a deciduous forest ecosystem, the presence of invasive Japanese stiltgrass (Microstegium vimineum) is used to infer decreased native plant diversity, as invasive plants in general are known to have that effect; (b) the density of the invasive shrub lantana (Lantana camara) is used to infer loss of native species due to established invasiveness-impact relationships and impact thresholds; (c) the ecological impacts of similar birds are used to infer those of the great kiskadee (Pitangus sulphuratus); and (d) niche models that predict the abundance of invasive common carp (Cyprinus carpio) are used to infer well-known waterquality effects of this benthic feeder.

relationship 3). This use of ecological surrogacy draws notable parallels to how surrogates are commonly applied in biodiversity monitoring, where particular taxa are monitored to provide information on a collection of unmeasured groups: that is, measuring a part to make inferences about the whole (Barton et al. 2015; Lindenmayer et al. 2015; Westgate et al. 2017). It also underpins the semi-quantitative categorical approach of classifying invasive species based on the magnitude of their largest demonstrated effect (eg as causing "minor", "moderate", or "major" impacts) that are becoming common in invasion science (Kumschick et al. 2012; Blackburn et al. 2014). Although it has been claimed that these classifications are based on adequate evidence, the contextual details of that evidence are generally ignored in subsequent research that considers the impact classification as a fixed and generalizable property of the species (Doherty et al. 2016; Evans et al. 2018). However, research on the impacts of invasive species is plagued by inconsistent methodology, and is largely restricted to snapshot studies that consider few response variables (Hulme et al. 2013). For example, two-thirds of invasive bird species that have been identified as having any impact were classified using evidence considered of "low-medium" confidence, meaning that all or some of the data are poor, difficult to interpret, and indirect (eg data are from another similar species) (Figure 2c; Evans et al. 2016). Using quantified impacts of a few metrics as surrogates for a broader impact assessment greatly underrepresents the variability of ecosystem effects that any species can have, which can be captured through the consistent testing of a larger variety of metrics (Barney et al. 2013).

\section{(4) Determinants of invasion as surrogates for consequences of invasion}

Assuming that invasion and invasiveness are strong predictors of invader impact, it stands to reason that the determinants of invasion can, in turn, act as surrogates for the consequences of invasion (Figure 1, relationship 4). This application essentially uses indirect measures of impact, meaning the measure is conceptually farther from the target than the other surrogateimpact relationships discussed above. Two or more links between 


\section{Panel 1. Using an adaptive surrogacy framework to select and evaluate surrogates for impact}

An adaptive surrogacy framework (Lindenmayer et al. 2015) treats proposed surrogates as working hypotheses to be subjected to rigorous testing. Although undertaking invasive species monitoring may not always be hypothesis driven (eg monitoring occurs for political, social, or economic motivations; Courchamp et al. 2017), using other measures to infer unmeasured ecological impact clearly represents a testable prediction based on assumptions and prior knowledge. Applying an adaptive framework to select and evaluate surrogates of ecological impact would make management practices more objective and remove bias from many of the ways invasive species are identified and prioritized for management across the globe. The key considerations of the framework in an invasion ecology context are detailed below.

\section{Identification of surrogates}

\section{Potential surrogates}

Consider all measurable qualities of a species invasion that could influence impact; they can include the presence of a species, some dimension of its invasiveness (eg local abundance), species traits and environmental context, and some aspect of the ecosystem that is likely to be impacted.

\section{Benchmarks and triggers}

It is important to establish clear reference points and baselines to assist later interpretation of the surrogate-target relationship; this consideration is critical for determining where ecosystem change relates to invader impacts or other influencing factors and natural variation, and ideally involves thorough understanding of the invaded ecosystem, timeseries monitoring, and control reference states.

\section{Sampling approach}

The methodology used to quantify a surrogate can greatly influence its accuracy and usefulness for representing impact; surrogate- impact relationships assessed at a particular spatial or temporal scale may not be transferable to a different spatial or temporal context. For instance, quantifying density effects of an invasive plant at a plot scale may not accurately represent impact at a site or landscape scale.

\section{Evaluation of surrogates}

\section{Scientific validity}

This is the critical step of quantifying the accuracy, certainty, and stability of the surrogate-impact relationship to be used. Although any surrogate for impact will be imperfect, it is important to quantify uncertainty and the level of confidence in the inferences being made. As such, the spatial and temporal boundaries under which the surrogate is robust and valid should be made clear.

\section{Cost effectiveness}

The monetary costs and benefits associated with invasive species monitoring, impact assessment, and surrogate identification and evaluation are not trivial and need to be considered. The most accurate surrogate for impact is unlikely to be monitored if it is very expensive to do so, whereas the most cost-effective surrogate may not be robust enough to confidently indicate impact and may instead be highly influenced by confounding factors.

\section{Risk assessment}

Consider and compare the range of possible ecosystem outcomes that may occur from either under- or overestimating ecological impact, and making decisions based on the "wrong" surrogate. For example, overestimating the impact of a species risks the misallocation of limited resources, whereas underestimating impact could have severe ecological consequences. surrogate and target create more opportunity for variation and error, meaning any inferences made will be inherently less accurate (Lindenmayer et al. 2015; Westgate et al. 2017). Although the determinants and consequences of invasion are largely considered to be independent of each other (Courchamp et al. 2017; Ricciardi et al. 2017), determinants of invasion, such as species traits and environmental context, are ultimately the most highly sought after by both researchers and practitioners to predict ecological consequences. For instance, nichebased modeling uses information on species traits and environmental tolerances to predict occurrence and abundance (ie invasion success), which are then used to infer impacts (Kulhanek et al. 2011). One study that employed this approach to predict invasive common carp (Cyprinus carpio) impacts recognized that predicted abundance was being used as a surrogate for impact (Kulhanek et al. 2011) but did not acknowledge that the niche model itself was using a surrogate for abundance, or that the contemporary ecosystem impacts of carp may be more nuanced (eg Kopf et al. 2017) than simply the long-established effects on water quality that the authors cited (Figure 2d). Similarly, for invasive birds, identifying specific traits that strongly predict impact may work in some instances (eg predatory birds that have major effects on native prey), but overall it is widely distributed generalist species that have the most severe (and diverse) impacts (Evans et al. 2018). The assumed strong links that justify using determinants of invasion as surrogates for impact are largely unsupported because the processes that determine invasion success are rarely the same as those that drive impact (Essl et al. 2017).

\section{Why the use of impact surrogates requires evaluation}

The use of surrogates is a practical means of facilitating evidencebased assessments where financial, temporal, or logistical constraints may limit collection of the most representative data (Lindenmayer et al. 2015; O'Loughlin et al. 2018). Surrogates 
are widely used to infer difficult-to-measure invasive species impacts for two key reasons. First, it is considered appropriate to assume that an invasive species will have severe ecological consequences so as to prioritize their management and avoid major impacts. Second, there are often practical constraints and ethical considerations that limit the ability of researchers and managers to quantitatively determine impacts in robust ways. Both approaches are motivated by the "precautionary principle", in that it is better to assume there will be negative impacts and act quickly than to wait for strong evidence and potentially risk irreversible change (Hulme et al. 2013). However, there are also potential risks associated with failing to evaluate the accuracy, stability, and certainty of surrogate-target relationships on a regular basis (Panel 1; Lindenmayer et al. 2015; Barton et al. 2015; Hunter et al. 2016).

Inferences derived from surrogate measurements involve unavoidable uncertainty, meaning there is a risk that the inference being made is wrong (O'Loughlin et al. 2018). The ways surrogates are currently used to infer ecological impacts of invasive species (Figure 1) represent practical decisions to measure something that is more generalizable, cost-effective, and communicable over something with greater accuracy, certainty, and robustness (ie the direct quantification of ecosystem effects; Lindenmayer et al. 2015). For instance, invasive mammalian predators are considered particularly damaging to native biodiversity, and have been implicated in the majority of documented vertebrate extinctions globally (Doherty et al. 2016). Using a more easily measured surrogate (eg presence or abundance of the predator) to infer population-level effects on native prey species is then justified given the potential for impact, limited conservation funding, and the need to communicate the issue to a broad and diverse audience (Courchamp et al. 2017). However, in a particular context, native prey populations may be far more responsive to habitat availability, disturbance history, or interspecific competition than an invasive predator (Zavaleta et al. 2001; Hradsky et al. 2017). For example, Hradsky et al. (2017) found that the relationship between invasive red fox (Vulpes vulpes) abundance and impact on native prey species was greatly influenced by fire and vegetation structure, suggesting measures of fox activity alone were poor surrogates for impact (Figure 3a). Simple and generalizable surrogates for biodiversity also show low consistency when context is considered (Westgate et al. 2017), highlighting that simple impact surrogates also may not be particularly robust.

Many researchers caution against using poorly assessed surrogate relationships to reach important conclusions and make decisions, given the high likelihood of chance correlation or context-dependency between a surrogate and target (Westgate et al. 2017; O'Loughlin et al. 2018). Similarly, high invasiveness of a species may merely reflect an altered or degraded community where the invader is a passenger and not a driver of change (Didham et al. 2005). For instance, the invasive giant African land snail (Achatina fulica) establishes and spreads within the rainforests on Christmas Island only after that ecosystem has been altered by other invasive taxa (O'Loughlin and
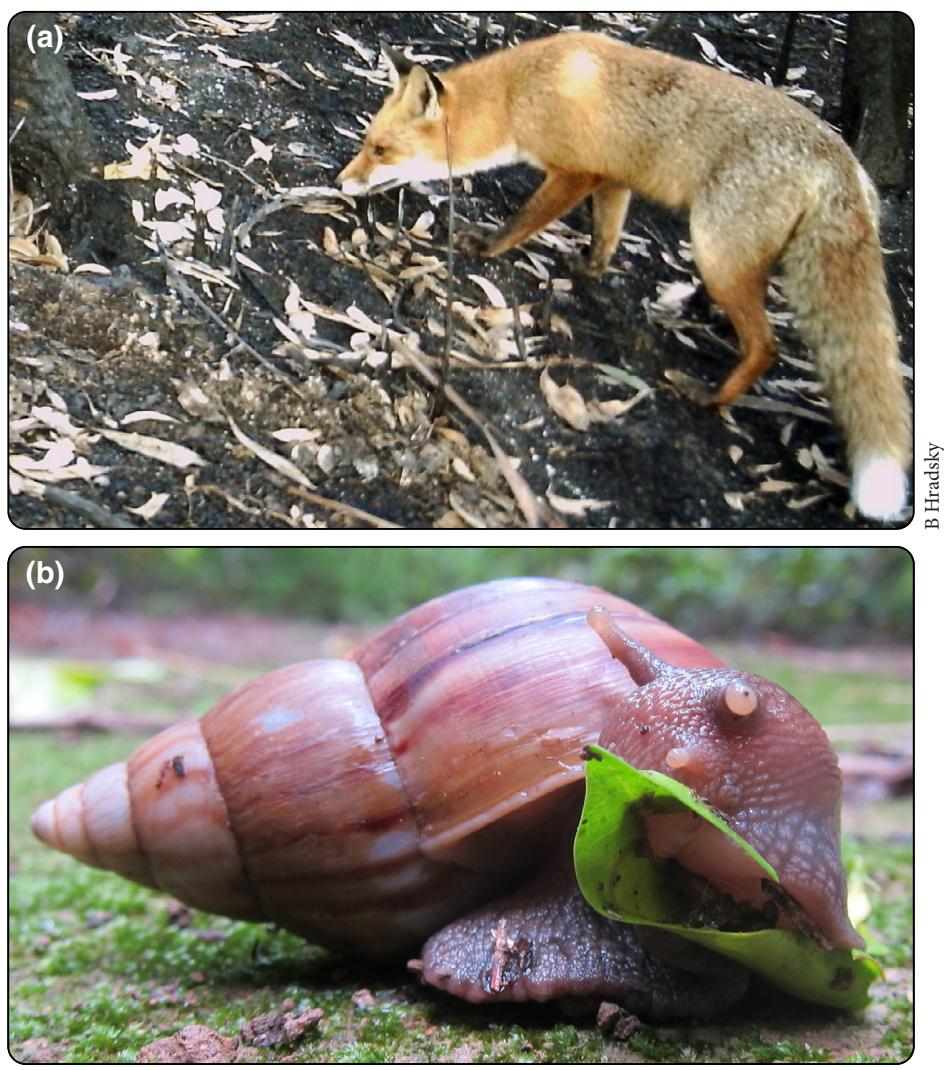

Figure 3. Examples of species for which the context of their invasion is critically important to understanding and inferring their ecological impacts. (a) Although invasive red foxes (Vulpes vulpes) have substantial impacts on native prey, often the magnitude of those impacts is mediated by disturbance and vegetation attributes (eg Hradsky et al. 2017), and as such red fox abundance alone may not be the most robust surrogate for impact. (b) The giant African land snail (Achatina fulica) is a problematic species throughout the tropics, yet on Christmas Island it is largely inhibited from establishing in rainforests and has limited impact when it does (0'Loughlin and Green 2017a), indicating that information about this species collected elsewhere was not a robust surrogate for its impacts on Christmas Island.

Green 2017b), and high densities of the snail do not have the measurable impacts on seedling recruitment or leaf litter dynamics that would be expected based on the snail's known traits and behaviors observed elsewhere (Figure 3b; O'Loughlin and Green 2017a). Therefore, any measure of this species' presence, abundance, functional traits, or impacts in a different setting would misrepresent the ecological impacts of this invasion.

The improved application of surrogates for impacts requires the adoption of a robust framework in which identified surrogates are explicitly evaluated for their scientific validity (see Panel 1). For example, Lindenmayer et al. (2015) developed an "adaptive surrogacy framework" that aims to unify surrogate concepts across disciplines and applications. Their framework is a guide to whether a surrogacy or direct measure approach is most effective in providing accurate information while also being cost-effective and 


\section{Surrogate validation model applied to invasive species management}

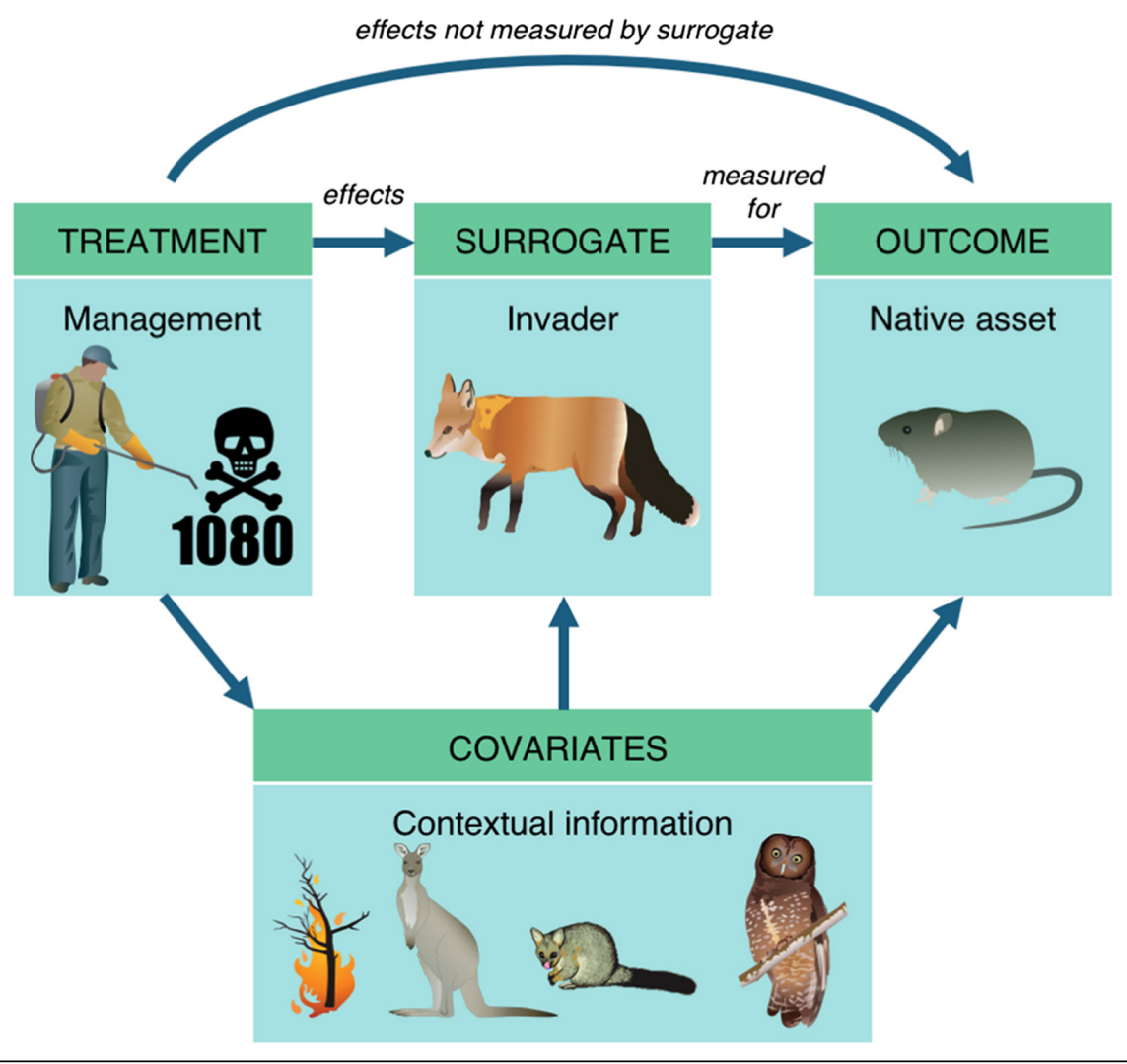

Figure 4. Framework for surrogate validation and its application in an invasive species management context. Conceptual model relates a treatment effect to a specified outcome via a surrogate (Panel 2). For invasive species management, measures of invasive species response to control efforts (ie management efficacy) are regularly used to infer positive ecosystem outcomes of that control. For example, the number of poison baiting stations deployed (treatment) is used to decrease the abundance of invasive predators (surrogate) in order to increase the population size of native prey species (outcome). There may also be direct (non-target) effects of the treatment on the outcome (eg number of prey species killed from poison baiting) and those effects may not be captured by measuring the surrogate. Abiotic drivers, competition, and native predators can influence the surrogate-outcome relationship (covariates) (Lindenmayer et al. 2018). Vector images are courtesy of the Integration and Application Network, University of Maryland Center for Environmental Science (www.ian.umces.edu/sym bols; used with permission).

easily communicated in a particular context (Panel 1). The key benefit to adopting this four-part hypothesis-driven framework in invasion science is that it provides researchers and managers with a tool to more clearly recognize their use of surrogates, to justify using them, and to avoid undue criticism when making inferences about impacts. Part 1 of the framework involves determining a clear target (that is specific, measurable, attainable, relevant, and time-bound), engaging with stakeholders to capitalize on diverse experiences and expertise, and developing a conceptual model of the target system to ensure links between potential surrogates and targets are logical; Parts 2 and 3 involve the identification and evaluation of surrogates, respectively (detailed in Panel 1); and Part 4 involves selecting and implementing a surrogate, and using active learning to identify key sources of uncertainty and continually improve the inferences being made. Greater integration of these kinds of lessons from surrogate ecology into invasion science offers a previously unrecognized solution to issues related to quantifying, demonstrating, managing, and communicating the ecological impacts of invasive species.

\section{Validating control of invasive species as a management surrogate}

The broad goals of conservation management are to control threats, and protect and enhance native ecosystem values. Therefore, considerable resources are dedicated to invasive species management programs under the premise that effective removal of an invasive species (a surrogate) corresponds to a reduction in a threat and some improvement to the invaded ecosystem (Figure 1, relationships 5 and 6, respectively) (Reid et al. 2009; Panetta et al. 2019). This approach reflects a conservation practitioner or manager applying the previously discussed surrogate-impact relationships to their program, and takes an additional step in logic by asserting that if invasion infers ecological impact, then removal of the invader removes the impact. Although that interpretation may seem overly simplified, in practice the link between invasiveness and impact is rarely evaluated before management is undertaken, and management effectiveness is typically determined only by observing the response of the invader and not broader ecosystem outcomes (Kettenring and Adams 2011; Doherty and Ritchie 2017; Prior et al. 2018). Similarly, when ecosystem recovery is measured following invasive species management, negative and mixed outcomes are almost as common as positive ones (Prior et al. 2018). The use of an invasive species' response to management or management itself (Figure 1, relationship 7) as a surrogate for benefits to the managed ecosystem may be greatly limited in instances where the link between invasiveness and impact was not first clearly determined.

There are many contrasting cases where successful management of an invader was or was not a good surrogate for positive ecosystem outcomes. For example, the eradication of invasive mammals from islands improves seabird nesting success and adult survival (Brooke et al. 2017), meaning the response of 


\section{Panel 2. Using causal frameworks to validate impact surrogates in response to management}

Causal frameworks examine the links between treatments and their effects, and how well surrogates can predict those effects (Figure 4; Barton et al. 2015). Whereas the presence and abundance of an invasive species may be a robust indicator of ecological impact, the removal of an invader through management intervention may not automatically indicate the removal of or recovery from those impacts. As such, the most accurate surrogate to infer ecological impact of an invader is unlikely to be the same surrogate that best represents ecological outcomes of management. This framework for surrogate validation should be used in combination with the adaptive framework for surrogate selection and evaluation (see Panel 1) to ensure the accuracy of inferred impacts of both invasive species and their management.

Surrogacy frameworks are toolkits for assessing the validity of claims with regard to invasive species impacts and management. For instance, invasive predators are frequently targeted for widespread control to protect native prey, yet the efficacy of this practice is rarely considered (see next paragraph for an example) (Doherty and Ritchie 2017). Therefore, when deciding whether to implement control actions and/or the reported outcomes of those actions, it is important to ask (1) How have predator impacts on native species and ecosystems been determined (ie what is the impact surrogate)? (2) How much evidence supports the assessment of impact (ie how robust is the impact surrogate)? (3) Have spatial and temporal covariates and environmental context been accounted for (ie how stable is the impact surrogate)? (4) Has management reduced predator impacts, not merely reduced predator numbers (ie how responsive is the impact surrogate)? and (5) How have positive biodiversity and ecosystem outcomes been shown to respond to management (ie how accurate is the impact surrogate)? The level of objectivity in the assessment of a species impact and the degree in which management action is evidence-based will become apparent by asking these questions of any program, and by explicitly considering the use of ecological surrogacy intrinsic to each of them.

For example, a poison baiting program to control invasive red fox (Vulpes vulpes) in an iconic Australian reserve was successful in terms of removing the invader, but unsuccessful in terms of having positive outcomes for native mammal fauna (Figure 4; Lindenmayer et al. 2018). Native mammals declined and some species became locally extinct over the 15 years following intense fox control (Figure 4). In this case (Lindenmayer et al. 2018), despite the treatment (baiting) having a major effect on the surrogate (fox numbers), the surrogate was not representative of the desired outcome (benefits to native prey). the invader to management is a strong indicator of a change in a threat (predation pressure) and positive outcomes for biodiversity (recovery of the impacted bird community). Conversely, removal of invasive plants more frequently leads to the establishment of other invaders, and cleared sites may show no evidence of return to the target native community even decades after removal, due to invader legacy effects and landscape disturbances (Maclean et al. 2018; Panetta et al. 2019). Similarly, native prey assemblages may collapse after the successful eradication of an invasive predator without any clear reason why (Lindenmayer et al. 2018). In those cases, information about the response of the invader to management alone is not an informative surrogate for either of the two broad goals of management (ie invader threat is not clear, and removal did not result in ecosystem recovery).

Ultimately, practitioners prioritize the management of invasive species based on the belief that those species are having severe ecological impacts, and for the most part lack the resources and capacity to test that assumption (Kuebbing and Simberloff 2015). However, failure to quantify the impacts of a species risks wasting limited resources on managing species that are not necessarily the most problematic, potentially leading to undesirable outcomes for the native ecosystem (Barney et al. 2013; Kopf et al. 2017). For instance, attempts to eradicate invasive cordgrass (Spartina spp) in California salt marshes led to population declines of endangered rails (Rallus spp) that were relying on cordgrass for nesting and foraging habitat (Lampert et al. 2014). These kinds of positive effects of invaders will be increasingly important for biodiversity in more modified ecosystems, and should be key considerations for scientists, managers, and decision makers prior to embarking on largescale control programs (Kopf et al. 2017). Whether or not the response of the invader to management action will be a robust surrogate for positive ecosystem outcomes will depend on the strength of the relationships between management treatment, surrogate measure, and conservation target, and how explicitly they are validated (Figure 4; Panel 2; Barton et al. 2015).

\section{Conclusions}

The threats posed by invasive species are underappreciated (Ricciardi and Ryan 2018); the frameworks and tools employed to assess invader impacts therefore need to be explicit and robust enough to limit misrepresentation and the risk of ineffective management (Courchamp et al. 2017). Possible solutions for improving impact assessments include (1) formally recognizing that ecological surrogacy is being implicitly used in invasive species research and management, and (2) applying quantitative approaches, used to evaluate and validate surrogate-target relationships in other fields, to surrogates of invasive species impacts. Surrogates are only as strong as the assumptions on which they are based, and support for many long-held assumptions in invasion ecology is declining (Jeschke et al. 2012). Inferring "impact" from "invasiveness" is a logical assumption, but an assumption nonetheless; therefore, it seems obvious that the accuracy, stability, and certainty of the surrogate-impact relationships that are widely used in invasion science would need to be rigorously and regularly tested. Current approaches for assessing the ecological impacts of invaders 
may downplay the uncertainty in measures and embrace a generalizable classification (eg Blackburn et al. 2014), advocate for greater direct quantitative assessments to improve accuracy (eg Barney et al. 2013), or strongly encourage more explicit measures of a species' per capita effects (eg Dick et al. 2017). For example, recent use of the "relative impact potential" metric (Dick et al. 2017) makes it clear that the measures like abundance and life span were being used as surrogates to infer population response (Dickey et al. 2018). This is an important step forward, and we suggest that greater appreciation of the frameworks already developed for assessing biodiversity surrogates (Lindenmayer et al. 2015) will further improve certainty around inferences of invader impacts through increased empirical quantification.

\section{Acknowledgements}

Constructive discussions with P Barton, J Catford, R Duncan, C Foster, P Green, A O'Loughlin, T O'Loughlin, D Panetta, C Sato, B Scheele, L Wandrag, and M Westgate greatly improved earlier versions of this article. The work was supported by an Australian Research Council Laureate Grant awarded to DBL.

\section{References}

Bacher S, Blackburn TM, Essl F, et al. 2018. Socio-economic impact classification of alien taxa (SEICAT). Methods Ecol Evol 9: 159-68.

Barney JN, Tekiela DR, Dollete ES, and Tomasek BJ. 2013. What is the "real" impact of invasive plant species? Front Ecol Environ 11: 322-29.

Barton PS, Pierson JC, Westgate MJ, et al. 2015. Learning from clinical medicine to improve the use of surrogates in ecology. Oikos 124: 391-98.

Blackburn TM, Essl F, Evans T, et al. 2014. A unified classification of alien species based on the magnitude of their environmental impacts. PLoS Biol 12: e1001850.

Blackburn TM, Pyšek P, Bacher S, et al. 2011. A proposed unified framework for biological invasions. Trends Ecol Evol 26: 333-39.

Brooke M de L, Bonnaud E, Dilley BJ, et al. 2017. Seabird population changes following mammal eradications on islands. Anim Conserv 21: 3-12.

Catford JA, Baumgartner JB, Vesk PA, et al. 2016. Disentangling the four demographic dimensions of species invasiveness. J Ecol 104: $1745-58$.

Catford JA, Jansson R, and Nilsson C. 2009. Reducing redundancy in invasion ecology by integrating hypotheses into a single theoretical framework. Divers Distrib 15: 22-40.

Courchamp F, Fournier A, Bellard C, et al. 2017. Invasion biology: specific problems and possible solutions. Trends Ecol Evol 32: $13-22$.

Dick JTA, Laverty C, Lennon JJ, et al. 2017. Invader relative impact potential: a new metric to understand and predict the ecological impacts of existing, emerging and future invasive alien species. $J$ Appl Ecol 54: 1259-67.
Dickey JWE, Cuthbert RN, Rea M, et al. 2018. Assessing the relative potential ecological impacts and invasion risks of emerging and future invasive alien species. NeoBiota 40: 1-24.

Didham RK, Tylianakis JM, Hutchison MA, et al. 2005. Are invasive species the drivers of ecological change? Trends Ecol Evol 20: 470-74.

Doherty TS, Glen AS, Nimmo DG, et al. 2016. Invasive predators and global biodiversity loss. P Natl Acad Sci USA 113: 11261-65.

Doherty TS and Ritchie EG. 2017. Stop jumping the gun: a call for evidence-based invasive predator management. Conserv Lett 10: $15-22$.

Essl F, Hulme PE, Jeschke JM, et al. 2017. Scientific and normative foundations for the valuation of alien-species impacts: thirteen core principles. BioScience 67: 166-78.

Evans T, Kumschick S, and Blackburn TM. 2016. Application of the Environmental Impact Classification for Alien Taxa (EICAT) to a global assessment of alien bird impacts. Divers Distrib 22: 919-31.

Evans T, Kumschick S, Şekercioğlu ÇH, and Blackburn TM. 2018. Identifying the factors that determine the severity and type of alien bird impacts. Divers Distrib 24: 800-10.

Gooden B, French K, Turner PJ, and Downey PO. 2009. Impact threshold for an alien plant invader, Lantana camara $\mathrm{L}$, on native plant communities. Biol Conserv 142: 2631-41.

Guerin GR, Martín-Forés I, Sparrow B, and Lowe AJ. 2018. The biodiversity impacts of non-native species should not be extrapolated from biased single-species studies. Biodivers Conserv 27: 785-90.

Hradsky BA, Mildwaters C, Ritchie EG, et al. 2017. Responses of invasive predators and native prey to a prescribed forest fire. $J$ Mammal 98: 835-47.

Hulme PE, Pyšek P, Jarošík V, et al. 2013. Bias and error in understanding plant invasion impacts. Trends Ecol Evol 28: 212-18.

Hunter M, Westgate M, Barton P, et al. 2016. Two roles for ecological surrogacy: indicator surrogates and management surrogates. Ecol Indic 63: 121-25.

Jarić I, Heger T, Monzon FC, et al. 2019. Crypticity in biological invasions. Trends Eco Evol 34: 291-302.

Jeschke JM, Bacher S, Blackburn TM, et al. 2014. Defining the impact of non-native species. Conserv Biol 28: 1188-94.

Jeschke J, Gómez Aparicio L, Haider S, et al. 2012. Support for major hypotheses in invasion biology is uneven and declining. NeoBiota 14: $1-20$.

Johnson AF and Lidström S. 2018. The balance between concepts and complexity in ecology. Nature Ecol Evol 2: 585-87.

Kettenring KM and Adams CR. 2011. Lessons learned from invasive plant control experiments: a systematic review and meta-analysis. J Appl Ecol 48: 970-79.

Kopf RK, Nimmo DG, Humphries P, et al. 2017. Confronting the risks of large-scale invasive species control. Nature Ecol Evol 1: 0172.

Kuebbing SE and Simberloff D. 2015. Missing the bandwagon: nonnative species impacts still concern managers. NeoBiota 25: 73-86.

Kulhanek SA, Leung B, and Ricciardi A. 2011. Using ecological niche models to predict the abundance and impact of invasive species: application to the common carp. Ecol Appl 21: 203-13.

Kumschick S, Bacher S, Dawson W, et al. 2012. A conceptual framework for prioritization of invasive alien species for management according to their impact. NeoBiota 15: 69-100. 
Lampert A, Hastings A, Grosholz ED, et al. 2014. Optimal approaches for balancing invasive species eradication and endangered species management. Science 344: 1028-31.

Lindenmayer D, Pierson J, Barton P, et al. 2015. A new framework for selecting environmental surrogates. Sci Total Environ 538: 1029-38.

Lindenmayer DB, Wood J, MacGregor C, et al. 2018. Conservation conundrums and the challenges of managing unexplained declines of multiple species. Biol Conserv 221: 279-92.

Lockwood JL, Cassey P, and Blackburn T. 2005. The role of propagule pressure in explaining species invasions. Trends Ecol Evol 20: 223-28.

Maclean JE, Mitchell RJ, Burslem DFRP, et al. 2018. Understorey plant community composition reflects invasion history decades after invasive Rhododendron has been removed. J Appl Ecol 55: 874-84.

Nentwig W, Bacher S, Pyšek P, et al. 2016. The Generic Impact Scoring System (GISS): a standardized tool to quantify the impacts of alien species. Environ Monit Assess 188: 315.

O'Loughlin LS and Green PT. 2017a. The secondary invasion of giant African land snail has little impact on litter or seedling dynamics in rainforest. Austral Ecol 42: 819-30.

O'Loughlin LS and Green PT. 2017b. Secondary invasion: when invasion success is contingent on other invaders altering the properties of recipient ecosystems. Ecol Evol 7: 7628-37.

O'Loughlin LS, Lindenmayer DB, Smith MD, et al. 2018. Surrogates underpin ecological understanding and practice. BioScience 68: 640-42.

Panetta FD and Gooden B. 2017. Managing for biodiversity: impact and action thresholds for invasive plants in natural ecosystems. NeoBiota 34: 53-66.

Panetta FD, O'Loughlin LS, and Gooden B. 2019. Identifying thresholds and ceilings in plant community recovery for optimal management of widespread weeds. NeoBiota 42: 1-18.

Parker IM, Simberloff D, Lonsdale WM, et al. 1999. Toward a framework for understanding the ecological effects of invader. Biol Invasions 1: 3-19.

Pearse IS, Sofaer HR, Zaya DN, and Spyreas G. 2019. Non-native plants have greater impacts because of differing per-capita effects and nonlinear abundance-impact curves. Ecol Lett 22: 1214-20.
Pearson DE, Ortega YK, Eren Ö, and Hierro JL. 2016. Quantifying "apparent" impact and distinguishing impact from invasiveness in multispecies plant invasions. Ecol Appl 26: 162-73.

Prior KM, Adams DC, Klepzig KD, and Hulcr J. 2018. When does invasive species removal lead to ecological recovery? Implications for management success. Biol Invasions 20: 267-83.

Pyšek P, Jarošík V, Hulme PE, et al. 2012. A global assessment of invasive plant impacts on resident species, communities and ecosystems: the interaction of impact measures, invading species' traits and environment. Glob Change Biol 18: 1725-37.

Reid AM, Morin L, Downey PO, et al. 2009. Does invasive plant management aid the restoration of natural ecosystems? Biol Conserv 142: 2342-49.

Ricciardi A and Cohen J. 2007. The invasiveness of an introduced species does not predict its impact. Biol Invasions 9: 309-15.

Ricciardi A and Ryan R. 2018. The exponential growth of invasive species denialism. Biol Invasions 20: 549-53.

Ricciardi A, Blackburn TM, Carlton JT, et al. 2017. Invasion science: a horizon scan of emerging challenges and opportunities. Trends Ecol Evol 32: 464-74.

Richardson DM, Pyšek P, Rejmánek M, et al. 2000. Naturalization and invasion of alien plants: concepts and definition. Divers Distrib 6: 93-107.

Seabloom EW, Borer ET, Buckley Y, et al. 2013. Predicting invasion in grassland ecosystems: is exotic dominance the real embarrassment of richness? Glob Change Biol 19: 3677-87.

Sofaer HR, Jarnevich CS, and Pearse IS. 2018. The relationship between invader abundance and impact. Ecosphere 9: e02415.

Spencer RJ, Van Dyke JU, and Thompson MB. 2016. The ethological trap: functional and numerical responses of highly efficient invasive predators driving prey extinctions. Ecol Appl 26: 1969-83.

Vilà M, Gallardo B, Preda C, et al. 2018. A review of impact assessment protocols of non-native plants. Biol Invasions 3: 1-15.

Westgate MJ, Tulloch AIT, Barton PS, et al. 2017. Optimal taxonomic groups for biodiversity assessment: a meta-analytic approach. Ecography 40: 539-48.

Zavaleta ES, Hobbs RJ, and Mooney HA. 2001. Viewing invasive species removal in a whole-ecosystem context. Trends Ecol Evol 16: 454-59. 